\title{
Direct comparison of FP-CIT SPECT and F-DOPA PET in patients with Parkinson's disease and healthy controls
}

\author{
S. A. Eshuis • P. L. Jager • R. P. Maguire • S. Jonkman • \\ R. A. Dierckx $\cdot$ K. L. Leenders
}

Received: 5 March 2008 / Accepted: 9 October 2008/Published online: 27 November 2008

(C) The Author(s) 2008. This article is published with open access at Springerlink.com

\begin{abstract}
Purpose Diagnosing Parkinson's disease (PD) on clinical grounds may be difficult, especially in the early stages of the disease. F-DOPA PET and FP-CIT SPECT scans are able to determine presynaptic dopaminergic activity in different ways. The aim of this study was to determine and compare the sensitivity and specificity of the two methods in the detection of striatal dopaminergic deficits in the same cohort of PD patients and healthy controls.

Methods Movement disorder specialists recruited 11 patients with early-stage PD and 17 patients with advanced PD. The patients underwent both an FP-CIT SPECT scan and an F-DOPA PET scan. In addition, 10 FP-CIT SPECT scans or 10 F-DOPA PET scans were performed in 20 healthy controls. A template with regions of interest was used to sample tracer activity of the caudate, putamen and a reference region in the brain. The outcome parameter was the striatooccipital ratio (SOR). Normal SOR values were determined in the controls. The sensitivity and specificity of both scanning methods were calculated.

Results FP-CIT SPECT and F-DOPA PET scans were both able to discriminate PD patients from healthy controls. For the early phases of the disease, sensitivity and specificity of the contralateral striatal and putaminal uptake of FP-CIT and F-DOPA was $100 \%$. When only caudate uptake was
\end{abstract}

S. A. Eshuis $(\square) \cdot$ R. P. Maguire $\cdot$ K. L. Leenders Department of Neurology, University Medical Center Groningen, University of Groningen,

P.O. Box 30001, 9700 RB Groningen, The Netherlands

e-mail: s.a.eshuis@ngmb.umcg.nl

P. L. Jager $\cdot$ S. Jonkman $\cdot$ R. A. Dierckx

Department of Nuclear Medicine and PET Centre,

University Medical Center Groningen, University of Groningen,

Groningen, The Netherlands considered, the specificities were $100 \%$ and $90 \%$ for FPCIT and F-DOPA, respectively, while the sensitivity was 91\% for both scanning techniques.

Conclusion FP-CIT SPECT and F-DOPA PET scans are both able to diagnose presynaptic dopaminergic deficits in early phases of PD with excellent sensitivity and specificity.

Keywords FP-CIT SPECT $\cdot$ F-DOPA PET.

Parkinson's disease $\cdot$ Diagnosis $\cdot$ Movement disorders

\section{Introduction}

Parkinson's disease (PD) is a neurodegenerative disorder, characterized by progressive loss of dopaminergic neurones in the substantia nigra. In the early stages of the disease it may be difficult to diagnose PD on the basis of clinical signs and symptoms. Diagnosing PD can also be hampered by the existence of nonneurodegenerative disorders, in which the presynaptic dopaminergic system is still intact, but which may mimic parkinsonism, such as essential tremor, vascular parkinsonism or drug-induced parkinsonism. A nonmovement disorder neurologist may misdiagnose up to $25 \%$ of patients with established parkinsonism of all sorts when compared with post-mortem pathology $[1,2]$. In contrast, movement disorder specialists can almost always correctly diagnose idiopathic PD when all the relevant clinical information is obtained. In such circumstances, the positive predictive value of the clinical diagnosis PD has been found to be $98.6 \%$ [3]. These studies relate to patients with advanced PD. No data are available to assess the situation in patients with early undiagnosed PD who on clinical grounds are indeed likely to have PD, but in whom this cannot yet be confirmed by response to medication or by the time course. This question is of even more interest for 
those patients in whom the clinician is not yet sure about the diagnosis, but would like to know whether a striatal dopaminergic defect is present or not [3]. Therefore, to facilitate diagnosing PD in the above-mentioned circumstances, auxiliary examinations are needed. For this purpose, conventional imaging techniques of the brain, such as CT and MRI scans, are not useful, because the brain structure is usually not greatly altered in early PD and dopaminergic biochemical activity cannot be positively documented by these techniques.

Radiotracer neuroimaging techniques using positron emission tomography (PET) or single photon emission computed tomography (SPECT) can be helpful in visualizing and measuring striatal dopaminergic activity $[4,5]$. PET scans using 6-[ $\left.{ }^{18} \mathrm{~F}\right]$ fluoro-L-3,4-dihydroxyphenylalanine (F-DOPA) enable measurement of striatal levodopa decarboxylase activity and trapping of F-dopamine in synaptic vesicles, which are decreased in PD [4-8]. It has been suggested that in the early stages of this disease, levodopa decarboxylase activity is upregulated $[9,10]$, although this has not yet been proved. Such factors, however, may influence the sensitivity of this technique for diagnosing defects in the nigrostriatal system.

Using SPECT, the uptake of tracers with a high affinity for the dopamine transporter (DAT) can be measured. DATs, located on dopaminergic nerve endings, participate in the reuptake mechanism of dopamine into presynaptic terminals and are modulated by concentrations of endogenous dopamine [11]. A decrease in DAT density in the striatum has been associated with PD $[12,13]$. DAT imaging can therefore be used as a marker for the degree of malfunction or loss of dopaminergic nerve endings. A selective and potent DAT imaging agent is $\left[{ }^{123} \mathrm{I}\right] N-\omega$-fluoropropyl-2 $\beta$ carbomethoxy-3 $\beta$-(4-iodophenyl) nortropane (FP-CIT). SPECT imaging with FP-CIT produces a high target to background ratio. Several studies have demonstrated that striatal FP-CIT uptake is reduced in patients with PD compared to healthy controls [14-22]. It has been shown that striatal uptake values of FP-CIT SPECT and F-DOPA PET correlate well with each other in patients with different stages of PD [15, 23].

It has been suggested that DATs are downregulated as an early response to reduction of the amount of endogenous dopamine. This would result in a decreased striatal binding of FP-CIT in early phases of PD. Therefore FP-CIT might be more sensitive than F-DOPA for detecting early striatal dopaminergic deficits.

The aim of this study was to determine and to compare intraindividually the sensitivity and specificity of the two tracer methods in the detection of striatal dopaminergic nerve terminal malfunctioning in the same cohort of PD patients and healthy controls.

\section{Materials and methods}

\section{Patients}

A total of 29 consecutive patients with strong clinical evidence of having PD were recruited between 2002 and 2003 from the Movement Disorders outpatient clinic of the University Hospital of Groningen, which has a tertiary referral function. Enrolment and clinical evaluation of participating patients was standardized and carried out by experienced movement disorder specialists. At clinical follow-up after more than 1 year after the two scans, 28 of the patients were still diagnosed with PD, confirmed by a documented response on symptomatic antiparkinson medication or, in drug-naive patients at follow up, worsening of extrapyramidal signs without any other symptoms. One patient did not respond to symptomatic antiparkinson medication and did not show any worsening of extrapyramidal signs after a period of 3 years of clinical follow-up. This patient was therefore excluded from further analyses.

The patients were divided into two groups: 11 patients $(8$ men and 3 women) with early-stage PD and 17 patients (15 men and 2 women) with advanced PD. The 11 patients with early-stage PD showed strong clinical evidence of PD based on two of the three cardinal symptoms (rest tremor, bradykinesia and rigidity), had a disease duration of less than 3 years, were not using antiparkinson medication, and had a Hoehn \& Yahr (H\&Y) stage of less than 2.0. The 17 patients with advanced PD showed two of the three cardinal symptoms, had a disease duration of 5-15 years, were using antiparkinson medication with a documented response, and had a H\&Y stage of 2.0-4.0. Patients with atypical signs, psychiatric disorders, signs of severe cognitive deterioration, severe cardiovascular comorbidity or on medication known to interfere with the DAT or catechol $O$-methyltransferase inhibitors, were excluded from the study.

A total of 20 healthy controls, 11 men and 9 women, in the same age range as the patients, were recruited. Subjects with psychiatric disorders, signs of severe cognitive deterioration, severe cardiovascular comorbidity or on medication known to interfere with the DAT, were excluded from the study.

Each patient with PD underwent a $\left[{ }^{123}\right.$ I]FP-CIT SPECT scan and a one-time-frame static F-DOPA PET scan. Ten healthy controls underwent a $\left[{ }^{123} \mathrm{I}\right] \mathrm{FP}-\mathrm{CIT}$ SPECT scan and ten others underwent a static F-DOPA PET scan. Written informed consent was obtained according to the Helsinki Convention and the study was approved by the local medical ethics committee. The ethics committee did not allow performance of both F-DOPA and FP-CIT scans in the same healthy control subjects. 


\section{F-DOPA PET scans}

Subjects fasted for at least $4 \mathrm{~h}$ before the start of the scan. Patients were allowed to continue their antiparkinson medication. On arrival subjects were given carbidopa $(2.5 \mathrm{mg} / \mathrm{kg})$ orally, and $60 \mathrm{~min}$ after the carbidopa dose, received $200 \mathrm{MBq}$ of F-DOPA intravenously. F-DOPA was prepared in the radiochemical laboratory of the University Medical Center Groningen as described elsewhere [24]. After a further $90 \mathrm{~min}$ from administration of the tracer, the subject was positioned in the PET camera (Siemens HR+; Erlangen, Germany) in a comfortable head holder with the orbitomeatal line in a transverse plane and a one-time-frame 3-D acquisition of 6 min duration was performed according to the standard operating procedures protocol of the University Medical Center Groningen. In daily clinical practice, static scans are preferred to dynamic scans in patients with $\mathrm{PD}$ as dynamic scans take a long time to perform and are therefore inconvenient. For estimation of the diagnostic value in this patient group it was therefore decided to perform static and not dynamic scans. Furthermore, static F-DOPA PET scans are preferred for a correct comparison with (static) FP-CIT SPECT scans. Finally, it is suggested that the striatal-occipital ratio (SOR) determined from a static scan can be as accurate as kinetic parameters, such as the binding constant $\mathrm{K}(\mathrm{occ})$, from a dynamic scan [25]. Scan data were reconstructed using iterative methods (ordered subsets expectation maximization) and were corrected for attenuation using a separate ellipse algorithm, according to the standard operating procedure in our centre for clinical PET scans.

\section{FP-CIT SPECT scans}

Patients were allowed to continue all medication, including antiparkinson medication. Subjects were injected with $185 \mathrm{MBq}$ FP-CIT (DaTscan, obtained commercially from GE, Eindhoven, The Netherlands). No thyroid blocking was given. After $180 \mathrm{~min}$ a SPECT acquisition was performed using a dual head gamma camera (Multispect 2; Siemens, Hoffman Estates, IL) with a LEHR (low-energy high-resolution) collimator, $128 \times 128$ image matrix, zoom factor $1.23,40 \mathrm{~s}$ per view and $2 \times 64$ views. Data acquisition was in agreement with the Dutch National Guidelines and with the guidelines from the manufacturer. Acquisition time was approximately $45 \mathrm{~min}$. Images were acquired in a symmetric $15 \%$ energy window around the photopeak of ${ }^{123} \mathrm{I}$ at $159 \mathrm{keV}$. The system resolution was $12 \mathrm{~mm}$ FWHM at $10 \mathrm{~cm}$. Subjects were carefully positioned in the gamma camera in a special head-holder which allowed minimal rotational distance with the orbitomeatal axis in a transverse plane to avoid reorientation during reconstruction. Image data were reconstructed using filtered back-projection and a Butterworth (0.50/6) filter. No attenuation correction was performed. As the aim of our study was to compare the two scanning methods in daily clinical practice, reconstruction of data were performed according to the standard operating procedures in our centre.

Image analysis

Reconstructed PET and SPECT data were realigned [26] to the common coordinate system of the Stereotactic Brain Atlas of Talairach and Tournoux. This realignment was performed using standard linear brain normalization algorithms (SPM software, FIL, London, UK). An image analysis algorithm was used to remove skin uptake. A standard set of regions of interest (ROIs) was used to sample both the caudate and putamen and a nonspecific reference region in the occipital cortex.

Ratios of specific to non-specific binding (SOR) were calculated by dividing the striatal count density by the occipital count density. ROI size-weighted average uptake values of the caudate and putamen were used to calculate mean whole striatal binding. In patients, uptake values in the same half of the body as the dominant (and generally initial) side of motor symptoms were called ipsilateral uptake values and those opposite to that side were called contralateral uptake values.

\section{Statistical analysis}

Shapiro-Wilk test was used to examine the normality of the distributions of the parameters of the different datasets. Normal values were calculated as the mean of all healthy controls \pm 2 standard deviations (SD).

Mean values in subgroups were compared using Student's $t$ test, and $t$ tests were also used to determine if uptake values in male controls differed significantly from those in female controls. If values were not normally distributed, Wilcoxon's rank sum was used. One-sided $p$ values of less than 0.05 were considered statistical significant.

The results of the FP-CIT SPECT and F-DOPA PET scans were initially considered abnormal when the uptake values were less than the mean values minus $2 \mathrm{SD}$ of the healthy controls. Next receiver operating curves (ROC) were produced and the sensitivity and specificity of the two scanning techniques for discriminating patients with earlystage PD from healthy controls were calculated. Sensitivity and specificity of F-DOPA and FP-CIT scans were compared using McNemar test. For determining the correlation between uptake values and age, Pearson's product was used if the data were normally distributed and Spearman's rho if the data were not normally distributed. 


\section{Results}

Group characteristics

The mean age of the healthy controls was 60.8 years (SD 8.4, range 40-74 years), the mean age of the patients with earlystage PD was 51.3 years (SD 10.1, range 33-66 years), and the mean age of the patients with advanced PD was 64.5 years (SD 6.3, range 46-71 years; see Table 1). The ages of the two groups of healthy controls were similar ( $t$ test, $p=0.78$ ). The age of the healthy controls was not significantly different from the ages of the patients in both groups ( $t$ test, $p=0.42$ ). However, as expected, the age of patients with advanced disease was higher than the age of patients with early-stage disease ( $t$ test, $p=0.001)$. There was no significant difference in age between patients with advanced disease and healthy controls ( $t$ test, $p=0.36$ ).

The male:female ratio was similar in the two patient groups (chi-squared test; $p=0.30$ ). However, the male: female ratio was higher in patients than in controls (chisquared test; $p=0.04$; see Table 1 ).

\section{Group results}

In the control subjects, the mean SOR values for the caudate, putamen and striatum, respectively, were 3.16 (range 2.703.86, SD 0.38), 2.65 (range 2.39-3.06, SD 0.21) and 2.90 (range 2.74-3.28, SD 0.26) for FP-CIT, and 2.69 (range 2.32-3.17, SD 0.25), 2.70 (range 2.29-3.42, SD 0.30) and 2.69 (range 2.31-3.30, SD 0.27) for F-DOPA (see Table 2). $\mathrm{SOR}_{\mathrm{FP}-\mathrm{CIT}}$ and $\mathrm{SOR}_{\mathrm{F}-\mathrm{DOPA}}$ for the left versus right caudate and the left versus right putamen were identical in the control subjects (all $p>0.2$ ). Examples of F-DOPA PET scans and FP-CIT SPECT scans are shown in Fig. 1.

\section{Disease discrimination}

\section{Contralateral values}

The $\mathrm{SOR}_{\mathrm{FPCIT}}$ and $\mathrm{SOR}_{\mathrm{F}-\mathrm{DOPA}}$ values for the caudate, putamen and striatum contralateral to the symptomatic side were significantly reduced in patients with early-stage PD compared with controls ( $t$ test; $p<0.0002$ for all comparisons; see also Fig. 2). Ipsilateral uptake values were also significantly reduced ( $t$ test; SOR FP-CIT $<<0.001$ for all comparisons). The measured uptake values of both tracers for the contralateral putamen and striatum did not show any overlap between patients and controls. However, these groups could not be completely separated by means of the calculated $2 \times$ SD interval (above and below the mean) of SOR $_{\text {F-DOPA }}$ for the contralateral putamen and striatum (Table 2).

In patients with early-stage PD, sensitivity and specificity based on contralateral putaminal and striatal SOR was 100\% for FP-CIT SPECT and for F-DOPA PET (see Table 3). Sensitivity based on contralateral caudate uptake values was the same $(91 \%)$ for FP-CIT and F-DOPA, while specificity was $90 \%$ for F-DOPA and $100 \%$ for FP-CIT. The ROC showed area under the curve (AUC) values of $0.98,1.00$ and 1.00 for the contralateral caudate, putaminal and striatal values, respectively, of FP-CIT, and AUC values of $0.95,1.00$ and 1.00 for the contralateral caudate, putaminal and striatal values, respectively, of F-DOPA. For the whole group of patients with early and advanced stages of disease, the specificity and sensitivity were 1.00 for both contralateral putaminal and striatal $\mathrm{SOR}_{\mathrm{FP}-\mathrm{CIT}}$ and SOR $_{\text {F-DOPA }}$ (see Table 3). Also the AUC values of the ROC curves were equal for FP-CIT and for F-DOPA uptake for the contralateral putamen and striatum. There were no significant differences in sensitivity and specificity between the two scanning techniques (McNemar test).

\section{Ipsilateral values}

When the analysis of disease discrimination was restricted to patients with $H \& Y$ stage 1 (i.e. one-sided parkinsonism without axial problems; $n=4$ ) and controls, ipsilateral uptake values were also significantly reduced in patients, albeit to a lesser degree $\left(t\right.$ test: SOR $\mathrm{FP}_{\text {-CIT }} p=0.0007$ for the caudate, $p=0.0002$ for the putamen and $p<0.0001$ for the striatum; $\mathrm{SOR}_{\mathrm{F}-\mathrm{DOPA}} p=0.0141$ for the caudate, $p=0.0097$ for the putamen and $p=0.0091$ for the striatum).

Table 1 Subject characteristics

\begin{tabular}{|c|c|c|c|c|c|c|}
\hline & \multicolumn{2}{|l|}{ Controls } & \multicolumn{2}{|c|}{ Early-stage patients } & \multicolumn{2}{|c|}{ Advanced-stage patients } \\
\hline & FP-CIT & F-DOPA & FP-CIT & F-DOPA & FP-CIT & F-DOPA \\
\hline No. of subjects & 10 & 10 & 11 & 11 & 17 & 17 \\
\hline Men & 5 & 5 & 8 & 8 & 15 & 15 \\
\hline Women & 5 & 5 & 3 & 3 & 2 & 2 \\
\hline \multicolumn{7}{|l|}{ Age (years) } \\
\hline Mean & 60.2 & 61.4 & 51.3 & 51.3 & 64.5 & 64.5 \\
\hline SD & 8.7 & 8.5 & 10.1 & 10.1 & 6.3 & 6.3 \\
\hline
\end{tabular}


Table 2 Uptake values of healthy controls and PD patients

\begin{tabular}{|c|c|c|c|c|c|c|c|}
\hline \multirow[t]{2}{*}{ Subject group } & \multirow[t]{2}{*}{ Region } & \multicolumn{3}{|c|}{ FP-CIT } & \multicolumn{3}{|c|}{ F-DOPA } \\
\hline & & Mean & $\mathrm{SD}$ & $2 \times \mathrm{SD}$ interval & Mean & SD & $2 \times \mathrm{SD}$ interval \\
\hline \multirow[t]{3}{*}{ Controls $(n=2 \times 10)$} & Mean caudate & 3.16 & 0.38 & $2.54-3.78$ & 2.69 & 0.25 & $2.19-3.19$ \\
\hline & Mean putamen & 2.65 & 0.21 & $2.19-3.11$ & 2.70 & 0.30 & $2.10-3.30$ \\
\hline & Mean striatum & 2.90 & 0.26 & $2.50-3.10$ & 2.69 & 0.27 & $2.15-3.23$ \\
\hline \multirow[t]{3}{*}{ Early-stage $(n=11)$} & Contralateral caudate & 2.22 & 0.28 & $1.66-2.78$ & 2.27 & 0.15 & $1.97-2.57$ \\
\hline & Contralateral putamen & 1.82 & 0.16 & $1.50-2.14$ & 1.91 & 0.15 & $1.61-2.21$ \\
\hline & Contralateral striatum & 2.02 & 0.17 & $1.68-2.36$ & 2.09 & 0.14 & $1.81-2.37$ \\
\hline \multirow[t]{3}{*}{ Advanced $(n=17)$} & Contralateral caudate & 1.91 & 0.47 & $0.97-2.85$ & 2.05 & 0.21 & $1.63-2.47$ \\
\hline & Contralateral putamen & 1.60 & 0.21 & $1.18-2.02$ & 1.76 & 0.11 & $1.54-1.98$ \\
\hline & Contralateral striatum & 1.76 & 0.32 & $1.12-2.40$ & 1.91 & 0.15 & $1.61-2.21$ \\
\hline
\end{tabular}

\section{Normal ageing}

In the group of 20 controls ( $n=10$ for each tracer), we found a significant age-dependent decline in SOR $\mathrm{FP}_{\mathrm{F}-\mathrm{CIT}}$ of $4.6 \%$ per decade for the mean putamen and of $3.9 \%$ per decade for the mean striatum $(p=0.04$ and 0.05 , respectively), but not for the mean caudate $(p=0.33)$. No significant effect of ageing was found for uptake values of
F-DOPA ( $p=0.75$ for the mean caudate, $p=0.65$ for the mean putamen and $p=0.92$ for the mean striatum).

\section{Gender differences}

In the group with healthy controls we found no significant effect of gender on striatal uptake of FP-CIT ( $t$ test: $p=0.77$ for the caudate, $p=0.08$ for the putamen and $p=0.50$ for the
Fig. 1 F-DOPA PET scans in (a) healthy controls and (b) patients with early-stage $\mathrm{PD}$, and FP-CIT SPECT scans in (c) healthy controls and (d) patients with early-stage PD
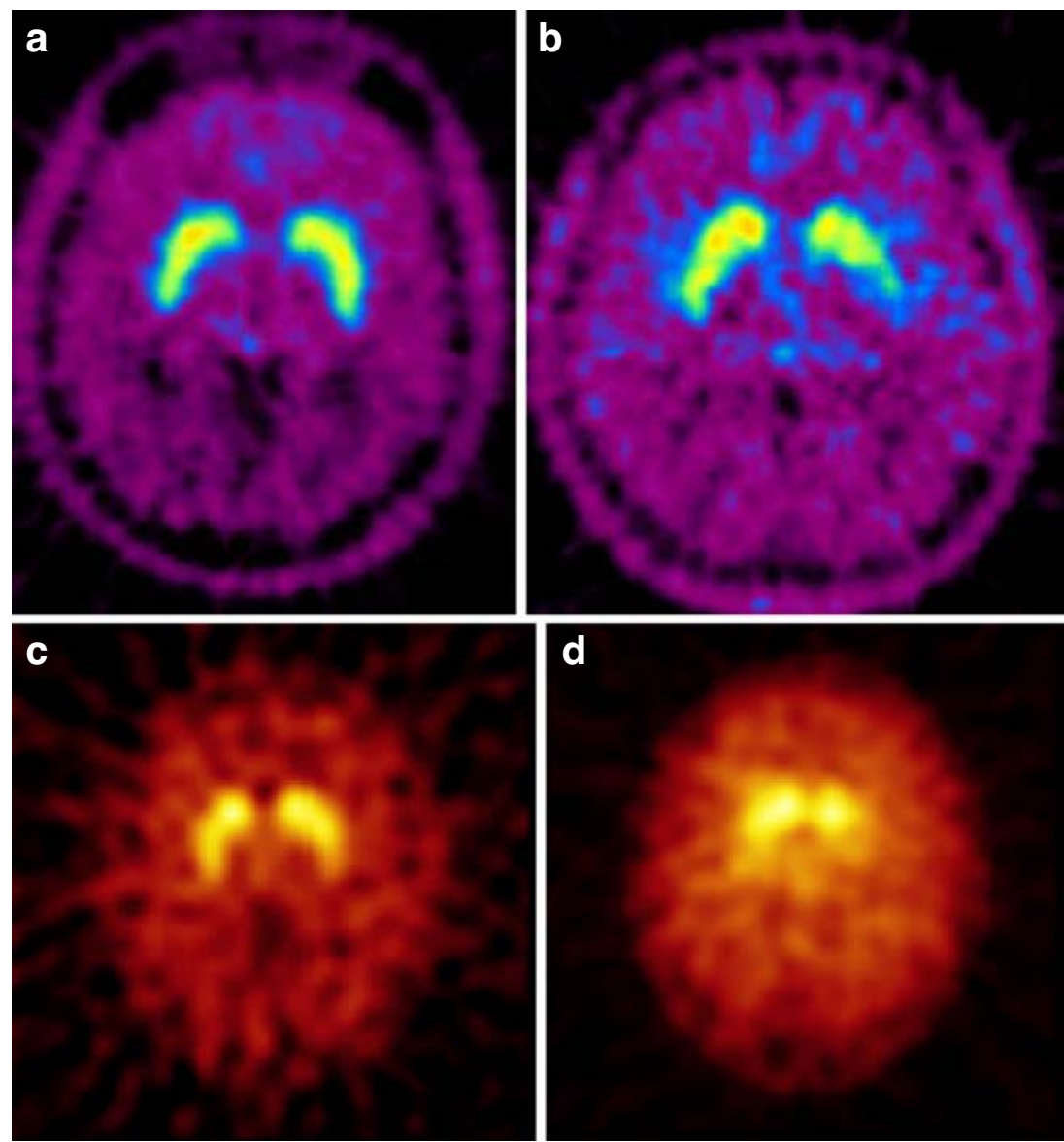

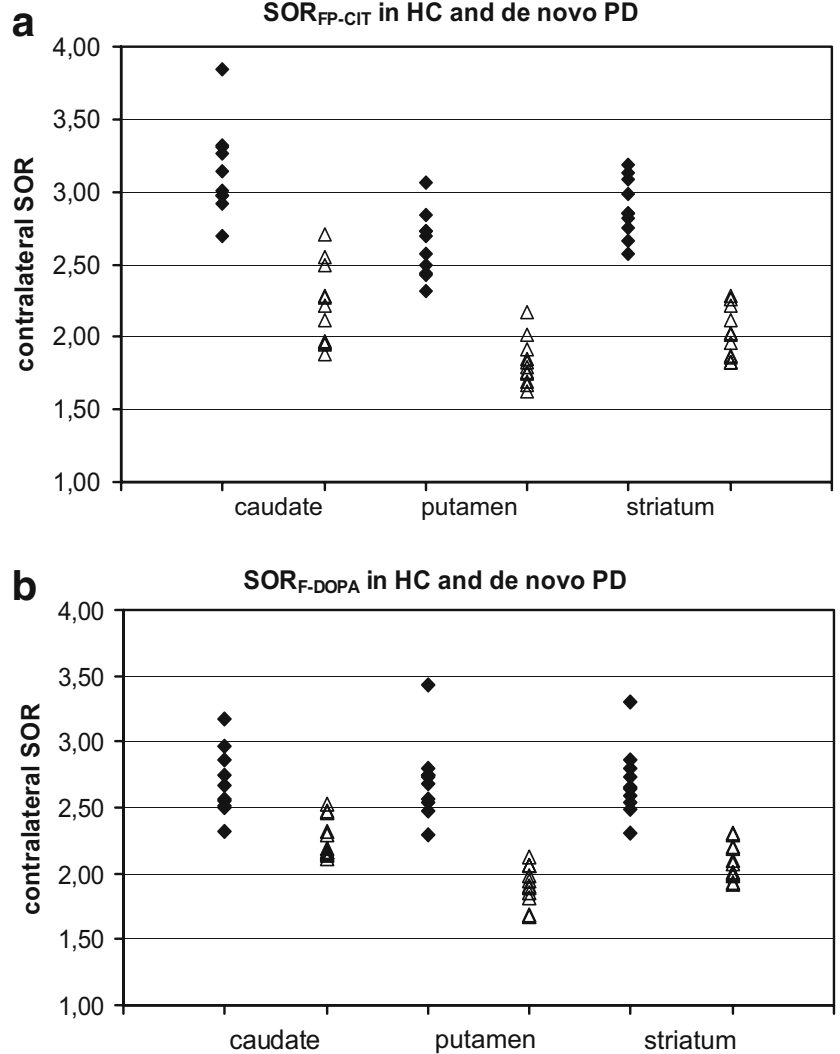

Fig. 2 Contralateral uptake values in healthy controls and patients with early-stage PD for (a) FP-CIT and (b) F-DOPA (closed diamonds healthy controls, open triangles patients with early-stage PD)

striatum). For F-DOPA, females tended to have slightly lower striatal uptake values than males. However, this difference did not reach statistical significance ( $t$ test: $p=$ 0.45 for the caudate, $p=0.12$ for the putamen and $p=0.23$ for the striatum).

\section{Discussion}

This study demonstrates that both FP-CIT SPECT and FDOPA PET scans are equally able to distinguish patients with PD patients from healthy controls. Our data show that scans with either tracer are able with $100 \%$ specificity and sensitivity to detect nigrostriatal damage in a group of patients with typical early-phase PD when considering tracer uptake in the contralateral putamen or striatum.

To our knowledge only one previous study has compared FP-CIT SPECT scans with F-DOPA PET scans in patients with mild PD and healthy volunteers [15]. With each method separately, they were able to identify correctly all 15 healthy controls and 11 out of 12 PD patients. Others reported a sensitivity of $95 \%$ to $97 \%$ for FP-CIT SPECT $[27,28]$. Morrish et al. were able to separate patients with PD from the normal group completely by F-DOPA PET [29]. Our findings emphasize the specificity of the applied methods. In our earlier study we demonstrated that both FPCIT SPECT and F-DOPA PET can be used to measure the presynaptic dopaminergic system in vivo and that they both show equally good ability to separate the early from the advanced stage of PD. Uptake values of F-DOPA correlated well with FP-CIT uptake values [23].

Both tracers are in vivo markers of the presynaptic dopaminergic system, but reflect different underlying biochemical mechanisms. F-DOPA uptake reflects the activity of aromatic L-amino acid decarboxylase (AADC) in the nerve terminals of the nigrostriatal dopaminergic pathway and the storage of F-dopamine in these nerve terminals $[4,5]$. FP-CIT binds to DATs, involved in the reuptake of dopamine from the synaptic cleft [11]. This difference between the two tracers results in a different method for examining the presynaptic dopaminergic system. In daily practice FP-CIT SPECT scanning is readily

Table 3 Sensitivity and specificity based on uptake values

\begin{tabular}{|c|c|c|c|c|c|c|c|}
\hline & \multirow[t]{2}{*}{ Region } & \multicolumn{3}{|l|}{ FP-CIT } & \multicolumn{3}{|l|}{ F-DOPA } \\
\hline & & Sensitivity (\%) & Specificity (\%) & AUC & Sensitivity (\%) & Specificity (\%) & AUC \\
\hline \multirow[t]{6}{*}{ Early-stage versus controls } & Contralateral caudate & 91 & 100 & 0.98 & 91 & 90 & 0.95 \\
\hline & Ipsilateral caudate & 82 & 90 & 0.86 & 64 & 90 & 0.80 \\
\hline & Contralateral putamen & 100 & 100 & 1.00 & 100 & 100 & 1.00 \\
\hline & Ipsilateral putamen & 100 & 100 & 1.00 & 91 & 90 & 0.95 \\
\hline & Contralateral striatum & 100 & 100 & 1.00 & 100 & 100 & 1.00 \\
\hline & Ipsilateral striatum & 73 & 100 & 0.95 & 82 & 90 & 0.92 \\
\hline \multirow[t]{6}{*}{ All patients versus controls } & Contralateral caudate & 100 & 91 & 0.98 & 90 & 96 & 0.98 \\
\hline & Ipsilateral caudate & 100 & 82 & 0.94 & 90 & 86 & 0.91 \\
\hline & Contralateral putamen & 100 & 100 & 1.00 & 100 & 100 & 1.00 \\
\hline & Ipsilateral putamen & 100 & 96 & 0.99 & 100 & 89 & 0.98 \\
\hline & Contralateral striatum & 100 & 100 & 1.00 & 100 & 100 & 1.00 \\
\hline & Ipsilateral striatum & 100 & 86 & 0.98 & 90 & 93 & 0.97 \\
\hline
\end{tabular}


applicable, in contrast to F-DOPA PET, whose use is limited by restricted availability of PET instruments and related tracer production. FP-CIT SPECT scanning, assuming that a normal dataset is available as reference for this technique, may therefore be the only realistic option to obtain relevant in vivo data about striatal dopaminergic activity. We found that in patients with likely early PD, a dopaminergic defect can be detected with FP-CIT SPECT scans as well as with F-DOPA PET scans. The finding of defects in the presynaptic dopaminergic system can be helpful for the physician to diagnose parkinsonian syndromes correctly in patients with doubtful clinical extrapyramidal symptoms.

Lee et al. [10] have examined the uptake of different tracers in patients with PD. They compared striatal uptake of $\left[{ }^{11} \mathrm{C}\right]$ methylphenidate (labelling DAT, comparable to the way FP-CIT does) with F-DOPA in patients with PD in an early phase of the disease. They found that in mildly affected parkinsonian patients, striatal F-DOPA uptake is relatively higher than the uptake of methylphenidate in the caudate as well as in the putamen. It was suggested that this difference in tracer uptake could be the result of an upregulation of AADC and a downregulation of the plasma membrane DAT in the striatum of patients with early PD. No direct proof of this has been obtained from human studies, although it is in line with experimental animal studies, which suggest a compensation of the loss of dopaminergic neurones by increasing the relative synthesis and release of dopamine from the remaining dopaminergic neurones [30, 31]. Ito et al. [9] concluded that in rats with early to advanced parkinsonism induced by 6-hydroxydopamine, $\left[{ }^{14} \mathrm{C}\right] \mathrm{L}$-DOPA levels underestimated the decrease in dopaminergic neurones and that $\left[{ }^{125} \mathrm{I}\right] \beta$-CIT levels more precisely reflected the decrease, suggesting that DAT is a better indicator of dopaminergic neuron loss. According to our data, striatal uptake of both tracers was reduced in patients with earlystage PD compared to healthy controls and no significant difference was found in disease discrimination between the two tracers.

We found that using either tracer, the striatal uptake in patients with unilateral PD (H\&Y I) was significantly reduced bilaterally without overlap of putamen values between PD patients and healthy controls. This implies that both methods can possibly also be used to identify patients in a preclinical phase of the disease. This has been confirmed by others $[16,18,21]$.

Values of healthy controls are needed to interpret correctly the results of functional neuroimaging. As different institutions may use different ROIs or different methods of analysis, each should obtain its own set of normal data. Our striatal uptake values of FP-CIT were higher than those of Innis et al. [32] and Tissingh et al., who found a striatal SOR in healthy controls of 2.24 and
2.43 [21]. Our caudate uptake values for FP-CIT were much higher than putaminal uptake values for FP-CIT (putamen:caudate ratio 0.83 for FP-CIT and 1.00 for FDOPA) in healthy controls. These high caudate uptake values were consistent through the complete set of data and could not be explained by outliers. Tissingh et al. found similar high caudate:putamen ratios [21]. Other studies have shown lower caudate uptake values in healthy controls. Also our SOR F-DOPA values were slightly higher than those of Ishikawa et al. who found uptake values of approximately $2.2[15,33]$.

One of the problems in evaluating neuroimaging methods in patients with early-stage PD is the validity of the clinical diagnosis of PD in this group. As mentioned before, diagnosing PD correctly based on clinical symptoms can be difficult and misdiagnosis occurs in up to $25 \%$ of patients in general practice. We have tried to overcome this problem by making the selection of participating patients by movement disorder specialists and by evaluating the clinical diagnosis after a period of more than 1 year after the performance of the scans.

We found a significant age-dependent decline in $\mathrm{SOR}_{\mathrm{FP}-\mathrm{CIT}}$ of $3.9 \%$ per decade in healthy controls, which is in line with the findings of others [15, 34], and in a post-mortem study a decay of $4.7 \%$ per decade of pigmented neurones in the substantia nigra was demonstrated [35]. It has been speculated that this ageing effect on DAT binding can be explained by compensation of neuronal dopamine loss by decreasing DAT synthesis [36]. Our study shows that the ageing effect on striatal DAT binding is relatively small and adjusting uptake values for age did not influence its accuracy for diagnosing presynaptic dopaminergic deficit. Also no significant effect of age on the symptomatic threshold of disease in PD was reported by others [15, 37].

In our data, striatal F-DOPA uptake did not change with age, although the number of subjects may have been too small to detect any ageing effect. This is in agreement with others $[15,38,39]$, although some studies have shown an ageing effect with F-DOPA PET [40, 41]. This doubtful effect of ageing on F-DOPA uptake may perhaps be explained by a lack of AADC decline during ageing [42], which is supported by post-mortem studies, indicating no decrease in AADC activity with age [43]. Another explanation may be that the remaining neurons produce less tyrosine hydroxylase (TH) per neuron resulting in a decrease in endogenous dopamine, while AADC and storage capacity for dopamine is maintained [44].

We found no significant effect of gender on striatal uptake values of either FP-CIT or F-DOPA in healthy controls. This is in agreement with some studies which also could not detect an effect of gender on FP-CIT uptake or FDOPA uptake [45, 46]. However, other studies have suggested that DAT tracer and F-DOPA uptake values are 
higher in women than in men [34, 47-49]. The lack of a significant effect of gender on striatal uptake values in our study may also be explained by the small number of participating healthy controls.

In this study we examined the uptake of two tracers in the same patients with clinically strong evidence of having $\mathrm{PD}$, also after a longer period of time. According to our study FP-CIT SPECT scans as well as F-DOPA PET scans are both able to detect early striatal dopaminergic defects and may therefore be of great help for the physician to diagnose PD correctly in an early phase of the disease. Patients with normal striatal uptake need to be reconsidered in terms of clinical condition. A person with clinical signs and symptoms which suggest parkinsonism but with normal striatal FP-CIT or F-DOPA uptake is not suffering from PD. However, it must be borne in mind that finding a striatal uptake reduction of either tracer does not provide by itself a clinical diagnosis of PD or other brain disease, but only demonstrates a striatal dopaminergic biochemical lesion.

\section{Conclusion}

Our study demonstrates that both FP-CIT SPECT scans and F-DOPA PET scans are able to diagnose presynaptic dopaminergic dysfunction and to separate patients with PD from healthy controls with high values of sensitivity and specificity.

Acknowledgements We thank GE Health for financial support.

The experiments complied with the current laws of the Netherlands, including approval of the local medical ethics committee.

Open Access This article is distributed under the terms of the Creative Commons Attribution Noncommercial License which permits any noncommercial use, distribution, and reproduction in any medium, provided the original author(s) and source are credited.

\section{References}

1. Hughes AJ, Daniel SE, Blankson S, Lees AJ. A clinicopathological study of 100 cases of Parkinson's disease. Arch Neurol 1993;50:140-8.

2. Meara J, Bhowmick BK, Hobson P. Accuracy of diagnosis in patients with presumed Parkinson's disease. Age Ageing 1999;28:99-102.

3. Hughes AJ, Daniel SE, Ben Shlomo Y, Lees AJ. The accuracy of diagnosis of parkinsonian syndromes in a specialist movement disorder service. Brain 2002;125:861-70.

4. Leenders KL, Palmer AJ, Quinn N, Clark JC, Firnau G, Garnett ES, et al. Brain dopamine metabolism in patients with Parkinson's disease measured with positron emission tomography. J Neurol Neurosurg Psychiatry 1986;49:853-60.

5. Leenders KL, Salmon EP, Tyrrell P, Perani D, Brooks DJ, Sager $\mathrm{H}$, et al. The nigrostriatal dopaminergic system assessed in vivo by positron emission tomography in healthy volunteer subjects and patients with Parkinson's disease. Arch Neurol 1990;47:1290-8.
6. Antonini A, Vontobel P, Psylla M, Günther I, Maguire PR, Missimer J, et al. Complementary positron emission tomographic studies of the striatal dopaminergic system in Parkinson's disease. Arch Neurol 1995;52:1183-90.

7. Garnett ES, Nahmias C, Firnau G. Central dopaminergic pathways in hemiparkinsonism examined by positron emission tomography. Can J Neurol Sci 1984;11:174-9.

8. Sawle GV, Playford ED, Burn DJ, Cunningham VJ, Brooks DJ. Separating Parkinson's disease from normality. Discriminant function analysis of fluorodopa $F 18$ positron emission tomography data. Arch Neurol1994;51:237-43.

9. Ito Y, Fujita M, Shimada S, Watanabe Y, Okada T, Kusuoka H, et al. Comparison between the decrease of dopamine transporter and that of L-DOPA uptake for detection of early to advanced stage of Parkinson's disease in animal models. Synapse 1999;31: $178-85$.

10. Lee CS, Samii A, Sossi V, Ruth TJ, Schulzer M, Holden JE, et al. In vivo positron emission tomographic evidence for compensatory changes in presynaptic dopaminergic nerve terminals in Parkinson's disease. Ann Neurol 2000;47:493-503.

11. Goodman MM, Keil R, Shoup TM, Eshima D, Eshima L, Kilts C, et al. Fluorine-18-FPCT: a PET radiotracer for imaging dopamine transporters. J Nucl Med 1997;38:119-26.

12. Kaufman MJ, Madras BK. Severe depletion of cocaine recognition sites associated with the dopamine transporter in Parkinsondiseased striatum. Synapse 1991;9:43-9.

13. Niznik HB, Fogel EF, Fassos FF, Seeman P. The dopamine transporter is absent in Parkinsonian putamen and reduced in the caudate nucleus. J Neurochem 1991;56:192-8.

14. Booij J, Tissingh G, Boer GJ, Speelman JD, Stoof JC, Janssen AG, et al. [123I]FP-CIT SPECT shows a pronounced decline of striatal dopamine transporter labelling in early and advanced Parkinson's disease. J Neurol Neurosurg Psychiatry 1997;62: 133-40.

15. Ishikawa T, Dhawan V, Kazumata K, Chaly T, Mandel F, Neumeyer $\mathrm{J}$, et al. Comparative nigrostriatal dopaminergic imaging with iodine-123-beta CIT- FP/SPECT and fluorine-18FDOPA/PET. J Nucl Med 1996;37:1760-5.

16. Kazumata K, Dhawan V, Chaly T, Antonini A, Margouleff C, Belakhlef A, et al. Dopamine transporter imaging with fluorine18-FPCIT and PET. J Nucl Med 1998;39:1521-30.

17. Marek K, Innis R, van Dyck C, Fussell B, Early M, Eberly S, et al. [123I]beta-CIT SPECT imaging assessment of the rate of Parkinson's disease progression. Neurology 2001;57:2089-94.

18. Marek KL, Seibyl JP, Zoghbi SS, Zea-Ponce Y, Baldwin RM, Fussell B, et al. [I-123] beta-CIT/SPECT imaging demonstrates bilateral loss of dopamine transporters in hemi-Parkinson's disease. Neurology 1996;46:231-7.

19. Seibyl JP, Marek KL, Quinlan D, Sheff K, Zoghbi H, Zea-Ponce $\mathrm{Y}$, et al. Decreased single-photon emission computed tomographic [123I]beta-CIT striatal uptake correlates with symptom severity in Parkinson's disease. Ann Neurol 1995;38:589-98.

20. Seibyl JP, Marek K, Sheff K, Zoghbi S, Baldwin RM, Charney DS, et al. Iodine-123-beta-CIT and iodine-123-FPCIT SPECT measurement of dopamine transporters in healthy subjects and Parkinson's patients. J Nucl Med 1998;39:1500-8.

21. Tissingh G, Booij J, Bergmans P, Winogrodzka A, Janssen AG, van Royen EA, et al. Iodine-123-N-omega-fluoropropyl-2betacarbomethoxy-3beta-(4-iodophenyl)tropane SPECT in healthy controls and early-stage, drug-naive Parkinson's disease. J Nucl Med 1998;39:1143-8.

22. Tissingh G, Bergmans P, Booij J, Winogrodzka A, van Royen EA, Stoof JC, et al. Drug-naive patients with Parkinson's disease in Hoehn and Yahr stages I and II show a bilateral decrease in striatal dopamine transporters as revealed by [123I]beta-CIT SPECT. J Neurol 1998;245:14-20. 
23. Eshuis SA, Maguire RP, Leenders KL, Jonkman S, Jager PL. Comparison of FP-CIT SPECT with F-DOPA PET in patients with de novo and advanced Parkinson's disease. Eur J Nucl Med Mol Imaging 2006;33:200-9.

24. de Vries EFJ, Luurtsema G, Brussermann M, Elsinga PH, Vaalburg W. Fully automated synthesis module for the high yield one-pot preparation of 6-[F-18]fluoro-L-DOPA. Appl Radiat Isotopes 1999;51:389-94.

25. Dhawan V, Ma Y, Pillai V, Spetsieris P, Chaly T, Belakhlef A, et al. Comparative analysis of striatal FDOPA uptake in Parkinson's disease: ratio method versus graphical approach. J Nucl Med 2002;43:1324-30.

26. Ashburner J, Friston K. Multimodal image coregistration and partitioning - a unified framework. Neuroimage 1997;6:209-17.

27. Benamer TS, Patterson J, Grosset DG, Booij J, de Bruin K, van Royen E, et al. Accurate differentiation of parkinsonism and essential tremor using visual assessment of [123I]-FP-CIT SPECT imaging: the [123I]-FP-CIT study group. Mov Disord 2000;15: 503-10.

28. Ortega Lozano SJ, Martinez Del Valle Torres MD, JimenezHoyuela Garcia JM, Gutierrez Cardo AL, Campos AV. Diagnostic accuracy of FP-CIT SPECT in patients with parkinsonism. Rev Esp Med Nucl 2007;26:277-85.

29. Morrish PK, Sawle GV, Brooks DJ. Clinical and [18F] dopa PET findings in early Parkinson's disease. J Neurol Neurosurg Psychiatry 1995;59:597-600.

30. Zigmond MJ, Berger TW, Grace AA, Stricker EM. Compensatory responses to nigrostriatal bundle injury. Studies with 6- hydroxydopamine in an animal model of parkinsonism. Mol Chem Neuropathol 1989;10:185-200.

31. Zigmond MJ, Abercrombie ED, Berger TW, Grace AA, Stricker EM. Compensations after lesions of central dopaminergic neurons: some clinical and basic implications. Trends Neurosci 1990;13:290-6.

32. Innis RB, Seibyl JP, Scanley BE, Laruelle M, Abi-Dargham A, Wallace E, et al. Single-photon emission computed tomographic imaging demonstrates loss of striatal dopamine transporters in Parkinson disease. Proc Natl Acad Sci U S A 1993;90:11965-9.

33. Ishikawa T, Dhawan V, Chaly T, Margouleff C, Robeson W, Dahl JR, et al. Clinical significance of striatal DOPA decarboxylase activity in Parkinson's disease. J Nucl Med 1996;37:216-22.

34. Lavalaye J, Booij J, Reneman L, Habraken JB, van Royen EA. Effect of age and gender on dopamine transporter imaging with [123I]FP-CIT SPET in healthy volunteers. Eur J Nucl Med 2000;27:867-9.

35. Fearnley JM, Lees AJ. Ageing and Parkinson's disease: substantia nigra regional selectivity. Brain 1991;114(Pt 5):2283-301.

36. Volkow ND, Ding YS, Fowler JS, Wang GJ, Logan J, Gatley SJ, et al. Dopamine transporters decrease with age. J Nucl Med 1996;37:554-9.
37. Booij J, Bergmans P, Winogrodzka A, Speelman JD, Wolters EC. Imaging of dopamine transporters with [123I]FP-CIT SPECT does not suggest a significant effect of age on the symptomatic threshold of disease in Parkinson's disease. Synapse 2001;39:101-8.

38. Eidelberg D, Takikawa S, Dhawan V, Chaly T, Robeson W, Dahl $\mathrm{R}$, et al. Striatal F-18 dopa uptake - absence of an aging effect. J Cereb Blood Flow Metab 1993;13:881-8.

39. Sawle GV, Colebatch JG, Shah A, Brooks DJ, Marsden CD, Frackowiak RS. Striatal function in normal aging - implications for Parkinson's disease. Ann Neurol 1990;28:799-804.

40. Martin WRW, Palmer MR, Patlak CS, Calne DB. Nigrostriatal function in humans studied with positron emission tomography. Ann Neurol 1989;26:535-42.

41. Vingerhoets FJ, Snow BJ, Schulzer M, Morrison S, Ruth TJ, Holden JE, et al. Reproducibility of fluorine-18-6-fluorodopa positron emission tomography in normal human subjects. J Nucl Med 1994;35:18-24.

42. Ota M, Yasuno F, Ito H, Seki C, Nozaki S, Asada T, et al. Agerelated decline of dopamine synthesis in the living human brain measured by positron emission tomography with L-[beta-C-11] DOPA. Life Sci 2006;79:730-6.

43. Kish SJ, Zhong XH, Hornykiewicz O, Haycock JW. Striatal 3,4dihydroxyphenylalanine decarboxylase in aging: disparity between postmortem and positron emission tomography studies? Ann Neurol 1995;38:260-4.

44. Haycock JW, Becker L, Ang L, Furukawa Y, Hornykiewicz O, Kish SJ. Marked disparity between age-related changes in dopamine and other presynaptic dopaminergic markers in human striatum. J Neurochem 2003;87:574-85.

45. Kaasinen V, Nurmi E, Bruck A, Bergman J, Solin O, Rinne JO. Increased frontal [F-18]fluorodopa uptake in early Parkinson's disease: sex differences in the prefrontal cortex. Brain 2001;124: 1125-30.

46. Ryding E, Lindstrom M, Bradvik B, Grabowski M, Bosson P, Träskman-Bendz L, et al. A new model for separation between brain dopamine and serotonin transporters in I-123-beta-CIT SPECT measurements: normal values and sex and age dependence. Eur J Nucl Med Mol Imaging 2004;31:1114-8.

47. Laakso A, Vilkman H, Bergman J, Haaparanta M, Solin O, Syvälahti E, et al. Sex differences in striatal presynaptic dopamine synthesis capacity in healthy subjects. Biol Psychiatry 2002;52: 759-63.

48. Staley JK, Krishnan-Sarin S, Zoghbi S, Tamagnan G, Fujita M, Seibyl JP, et al. Sex differences in [I-123]beta-CIT SPECT measures of dopamine and serotonin transporter availability in healthy smokers and nonsmokers. Synapse 2001;41:275-84.

49. Haaxma CA, Bloem BR, Borm GF, Oyen WJ, Leenders KL, Eshuis S, et al. Gender differences in Parkinson's disease. J Neurol Neurosurg Psychiatry 2007;78:819-24. 